ARTICLE

https://doi.org/10.1038/s41467-020-14441-7

\title{
Structural basis of proton-coupled potassium transport in the KUP family
}

Igor Tascón (1) 1,5, Joana S. Sousa ${ }^{2,5}$, Robin A. Corey³, Deryck J. Mills², David Griwatz (D) 1, Nadine Aumüller ${ }^{1}$, Vedrana Mikusevic ${ }^{1}$, Phillip J. Stansfeld (10 ${ }^{3,4}$, Janet Vonck ${ }^{2 \star} \&$ Inga Hänelt (1) ${ }^{1 \star}$

Potassium homeostasis is vital for all organisms, but is challenging in single-celled organisms like bacteria and yeast and immobile organisms like plants that constantly need to adapt to changing external conditions. KUP transporters facilitate potassium uptake by the cotransport of protons. Here, we uncover the molecular basis for transport in this widely distributed family. We identify the potassium importer KimA from Bacillus subtilis as a member of the KUP family, demonstrate that it functions as a $\mathrm{K}^{+} / \mathrm{H}^{+}$symporter and report a 3.7 A cryo-EM structure of the KimA homodimer in an inward-occluded, trans-inhibited conformation. By introducing point mutations, we identify key residues for potassium and proton binding, which are conserved among other KUP proteins.

\footnotetext{
${ }^{1}$ Institute of Biochemistry, Goethe University Frankfurt, Frankfurt am Main, Germany. ${ }^{2}$ Department of Structural Biology, Max Planck Institute of Biophysics, Frankfurt am Main, Germany. ${ }^{3}$ Department of Biochemistry, University of Oxford, Oxford, UK. ${ }^{4}$ School of Life Sciences \& Department of Chemistry, University of Warwick, Coventry CV4 7AL, UK. ${ }^{5}$ These authors contributed equally: Igor Tascón, Joana S. Sousa. *email: janet.vonck@biophys.mpg.de; Haenelt@biochem.uni-frankfurt.de
} 
K is the most abundant intracellular cation in all living organisms and its homoeostasis is absolutely essential. Microorganisms depend on a variety of different $\mathrm{K}^{+}$ uptake systems to adapt to rapidly changing external conditions. The main players here can be grouped in two families, the Trk/ $\mathrm{Ktr} / \mathrm{HKT} / \mathrm{Kdp}$ family ${ }^{1}$ and the Kup/HAK/KT (K+ Uptake, KUP) family ${ }^{2-4}$. Interestingly, members of both families are absent in mammalian cells, while they were identified as virulence factors in pathogenic bacteria ${ }^{5-8}$. The KUP family belongs to the APC (amino acid-polyamine-organocation) superfamily, the second largest superfamily of secondary active transporters ${ }^{9}$. Members of the KUP family have different physiological roles in diverse bacteria and even in different plant organs, with correspondingly a wide scope of affinities for $\mathrm{K}^{+}$, ranging from nano- to millimolar concentrations ${ }^{10-13}$. KUP proteins have been proposed to be particularly important under acidic environmental conditions and to function as $\mathrm{K}^{+} / \mathrm{H}^{+}$symporters ${ }^{14-16}$. Reporter fusions and cysteine labelling assays suggested the presence of 12 transmembrane helices and several residues crucial for $\mathrm{K}^{+}$transport activity have been identified in different KUP members ${ }^{11,17-20}$. Yet, due to the lack of detailed structural information KUPs' transport mechanism remains largely unknown.

Recently, KimA from Bacillus subtilis was identified as a highaffinity potassium importer ${ }^{21}$ and KimA homologs were proposed to form a family within the APC superfamily. Here, we show that KimA is in reality a member of the KUP family. We functionally characterized KimA in vivo and in vitro and solved a trans-inhibited, inward-occluded structure using cryo-EM. In combination with mutational analysis, we propose a coupling mechanism for the symport of potassium ions and protons.

\section{Results}

KimA functions as a $\mathbf{K}^{+} / \mathbf{H}^{+}$symporter. KimA was previously identified as a potassium uptake system in Bacillus subtilis cells depleted of its long-known potassium-importing channels, KtrAB and $\mathrm{KtrCD}^{21}$, and is wide-spread among bacteria (Supplementary Fig. 1). To characterize the $\mathrm{K}^{+}$uptake mode of KimA, we performed in vivo and in vitro transport assays. In vivo, KimA mediates potassium uptake into potassium-depleted Escherichia coli LB2003 cells deficient in endogenous potassium uptake systems, with a $K_{M}$ value of $215 \mu \mathrm{M}$ and a $V_{\max }$ of $245 \mathrm{nmol} \mathrm{min}^{-1}$ $\mathrm{mg}^{-1}$ (Fig. 1a, b). Potassium uptake increased at lower external $\mathrm{pH}$ and was abolished in the presence of the proton ionophore CCCP (Fig. 1c). The observed $\mathrm{pH}$ dependency and the apparent requirement for a proton gradient suggest that KimA functions as a $\mathrm{K}^{+} / \mathrm{H}^{+}$symporter. In vitro transport assays performed in proteoliposomes, in the presence of an inward-directed potassium
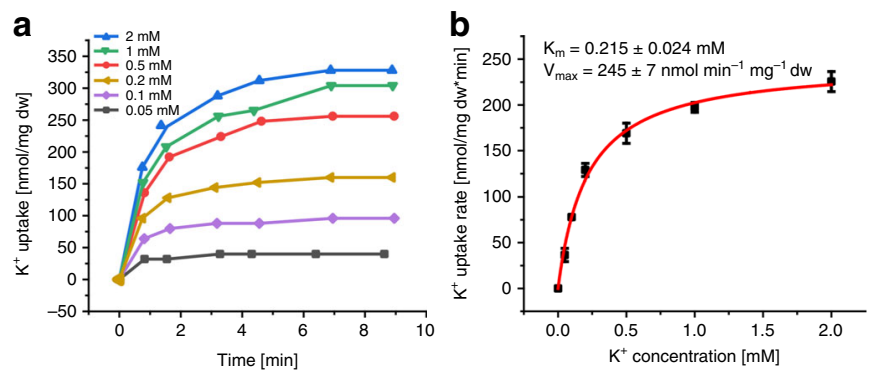

gradient, confirmed that KimA couples potassium uptake and proton transport (Fig. 1d).

Structure of dimeric KimA in inward-occluded conformation. To elucidate the structural basis for proton-coupled potassium transport, we determined a $3.7 \AA$ resolution cryo-EM structure of KimA solubilized with styrene-maleic acid (SMA) co-polymers in the presence of potassium (Fig. 2, Supplementary Figs. 2 and 3 and Supplementary Table 1). Our structure shows that KimA forms a homodimer, with each protomer consisting of an $\mathrm{N}$ terminal transmembrane (TM) domain and a C-terminal cytoplasmic domain (Fig. 2a, b), as previously suggested ${ }^{22}$. The dimer is stabilized by the swapping of the cytoplasmic domains, which are placed under the opposite membrane domains via long loops connected to the last TM helix (Fig. 2a). The interaction of these loops with the other cytoplasmic domain forms the main dimer interface (Fig. 2c). The two TM domains are tilted towards each other at the extracellular side, creating a second interface, and appear to enforce a bending of the membrane by $\sim 130^{\circ}$ against its natural curvature (Supplementary Fig. 4a). The same arrangement is observed for detergent-solubilized KimA (Supplementary Fig. $4 \mathrm{~b}$ and $\mathrm{c}$ ), demonstrating that this shape does not derive from forces exerted by the SMA co-polymer over the protein. However, we could not exclude that the depletion of lipids from the dimer interspace during purification may have led to the observed interface. To investigate this further, we carried out molecular dynamics (MD) simulations on the KimA dimer. An initial 100 ns coarse-grained $(\mathrm{CG})^{23,24}$ simulation on the positionallyrestrained KimA dimer reveals that the membrane freely forms around the dimer (Fig. 3a; 'input'). This results in a substantial bending of the bilayer, in a similar arrangement to the one observed in the EM map. When investigating the dynamics of the unrestrained dimer over a total of $20 \mu$ s, we see that it relaxes into an 'upright dimer' arrangement with the TM domains separated (Fig. 3a). The dimer switches between both positions in a highly dynamic fashion (Supplementary Fig. 5a), with each arrangement about equally likely. Plotting the angle of each TM domain over the course of the simulation reveals that the two monomers move in concert, akin to a 'breathing' motion (Fig. 3b, Supplementary Fig. 5b, c). Such 'breathing' motion was also observed in the EM sample during the refinement, albeit to a lesser extent. Upright dimers were not observed in the cryo-EM analysis. Follow-up simulations totalling $6 \mu$ s using the atomistic CHARMM36 force field confirm the presence of the upright dimer, with $2 / 3$ systems adopting the same pose (Fig. $3 \mathrm{c}$ and Supplementary Fig. 5d) and principal component analysis (PCA) on the data revealing that the breathing motion accounts for $68 \%$ of the total variance (Supplementary Fig. 5e). These MD simulations reveal that
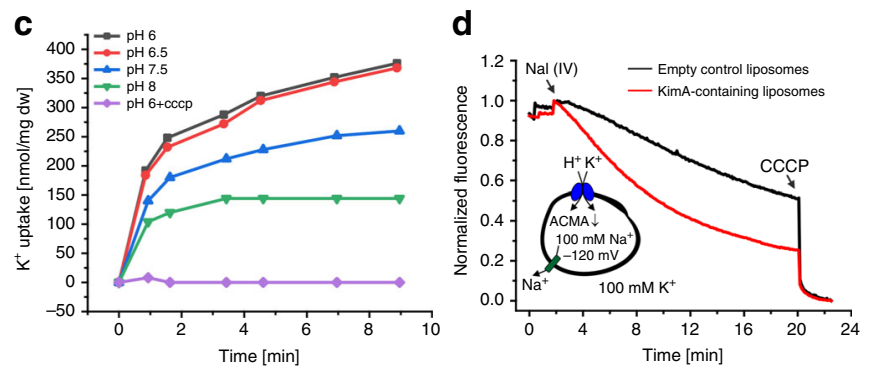

Fig. 1 Functional analysis of KimA. a Concentration-dependent potassium uptake via KimA into potassium-depleted $E$. coli LB2003 cells. $n=3$ independent experiments; a representative experiment is shown. b. Kinetic parameters of the potassium transport via KimA determined based on (a). The plotted uptake rates at different potassium concentrations are the means of three independent experiments; errors shown are s.d. c pH-dependent potassium transport via KimA into potassium-depleted E. coli LB2003. $n=3$ independent experiments; a representative experiment is shown. $\mathbf{d}$ Potassiumdependent proton transport into KimA-containing liposomes. $n=3$ independent experiments; a representative experiment is shown. Source data are provided as a Source Data file. 


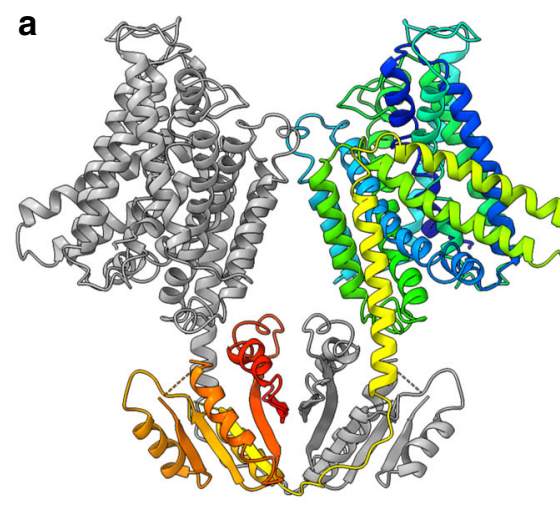

C

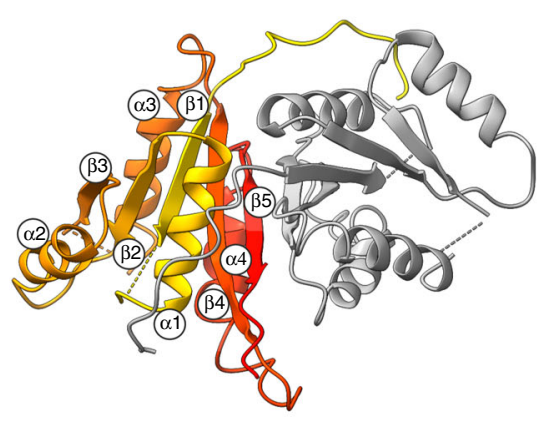

b

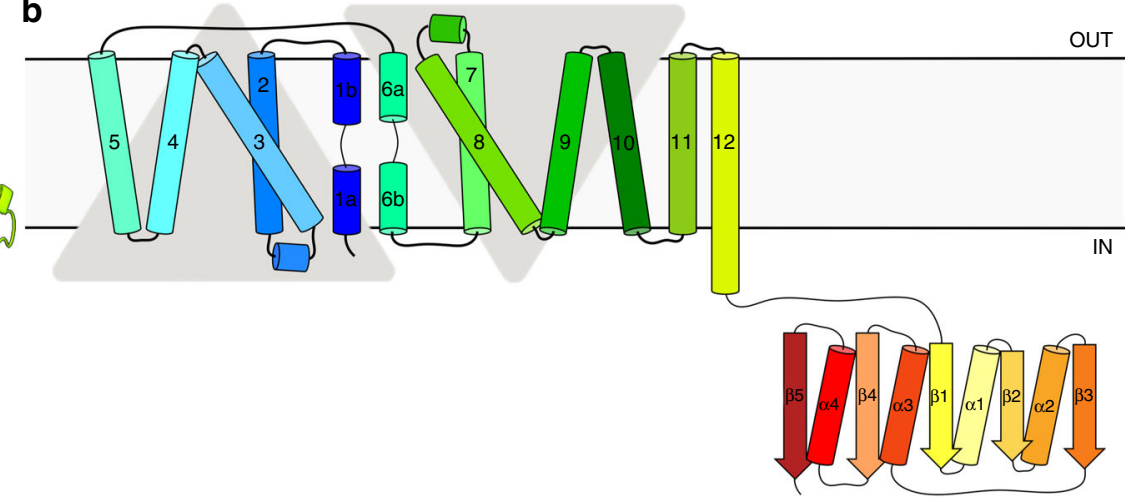

d

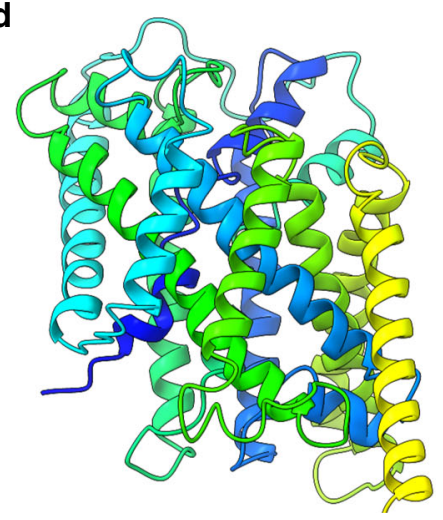

e

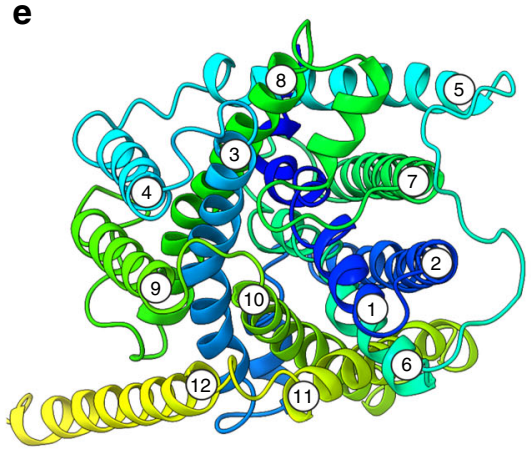

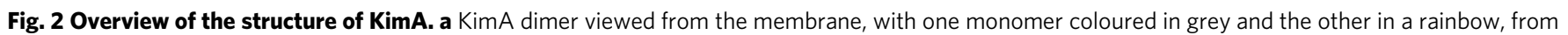

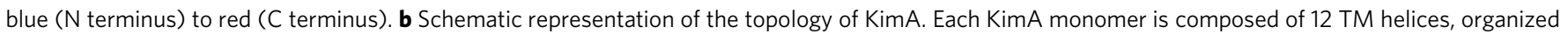

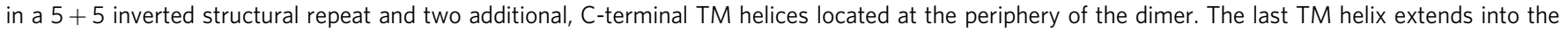

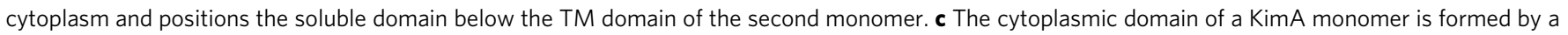
parallel, five-stranded $\beta$-sheet sandwiched by four $\alpha$-helices, forming a continuous 10 -stranded $\beta$-sheet in the dimer. $\mathbf{d}$ Side view and e top view of the membrane domain of a KimA monomer.

approximately twice as many lipids bind in between the protomers in the upright dimer compared to the tilted dimer (Fig. 3d). We speculate that not enough lipids were present after purification to fill the inter-dimer space and stabilize the upright position of the TM domains. Importantly, the inter-subunit movement did not affect the conformation of the TM domains over the course of the performed simulations. It remains elusive whether this movement has any physiological relevance or functional consequences.

The cytoplasmic domain of each protomer consists of $\beta$ strand- $\alpha$-helix motifs, which form a parallel 5 -stranded $\beta$-sheet flanked by four helices and in the dimer generate an extended $\beta$ sheet with 10 strands (Fig. 2a, c). Interestingly, the same fold was previously observed in a number of proteins, including soluble phosphopantetheine adenylyltransferases (PPAT) (PDB 1GN8) ${ }^{25}$, which bind ATP and ADP (Supplementary Fig. 7), and the cytoplasmic domain of a prokaryotic cation-chloride cotransporter (PDB 3G40)26. In analogy to the nucleotide binding to PPAT, the cytoplasmic domains of KimA may provide binding sites for cyclic di-AMP, which was recently identified as an inhibitor for the potassium uptake by KimA ${ }^{27}$. However, although $50 \mu \mathrm{M} \mathrm{c}$-diAMP was added to purified KimA before sample freezing, no additional density for the molecule was observed, suggesting that it could not bind to the present state. In agreement to this assumption, no c-di-AMP binding could be determined using isothermal titration calorimetry and thermal shift assay (data not shown).

Each TM domain contains 12 TM helices (Fig. 2b, d, e), the first ten of which adopt a typical LeuT fold ${ }^{28}$, with a topologically inverted repeat of TM helices 1-5 and TM helices 6-10 and broken helices 1 and 6 (Supplementary Fig. 8). TM helix 12 extends into the cytoplasm (Fig. 2) and, as previously mentioned, connects to the cytoplasmic domain. The structure represents an inward-occluded conformation of KimA. The extracellular side is tightly sealed by main chain packing of TM helices $1 \mathrm{~b}, 3$, 6a, and 10 , while on the cytoplasmic side we observe a wide solvent-filled tunnel, lined by polar residues (D117, Y118, E233, N237, T317, S320, Q324) (Fig. 4a, b). A thin gate ${ }^{29}$, formed by the side chains of D36, T121, T230 and Y377, separates this tunnel from a smaller cavity located next to the discontinuous region of TM helices 1 and 6 , which we hypothesize to be a potassium ion binding site (Fig. 4a, b).

Each TM domain has three potassium ions bound. In agreement with the hypothesized potassium ion binding site, the cryoEM map shows a non-protein density in this region, between D36, Y43, T121, S125, T230, and Y377, consistent with the presence of a potassium ion (Fig. 4c, d, Supplementary Fig. 3f). This location corresponds to the substrate binding site in other transporters with the LeuT fold.

Below the thin gate two other non-protein densities, separated by $\sim 3 \AA$, are observed near the side chains of D36, D117, and E233 (Fig. 4e and Supplementary Fig. 3g). As the buffer contained $100 \mathrm{mM}$ potassium chloride, and smaller molecules like waters are only visible at a resolution better than $3 \AA$, we also assign these densities as potassium ions (Fig. 4c-e). Their close proximity may indicate that their positions are not occupied at the same time and the map densities have been averaged. 
a
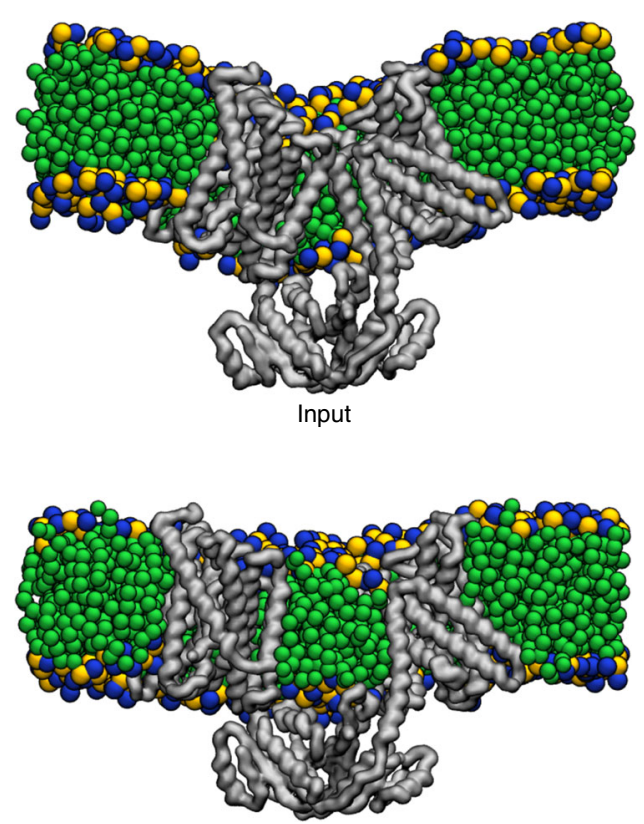

Upright dimer

d

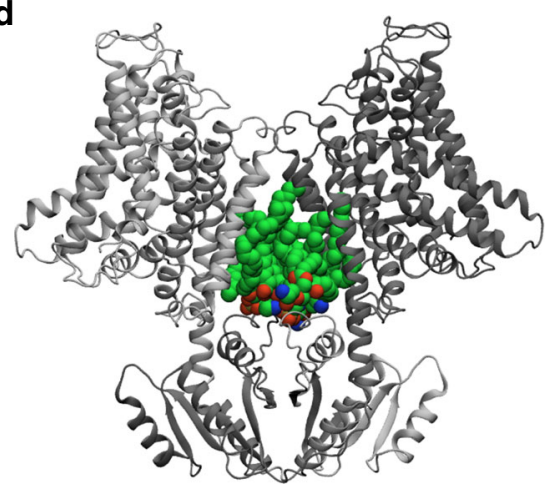

b

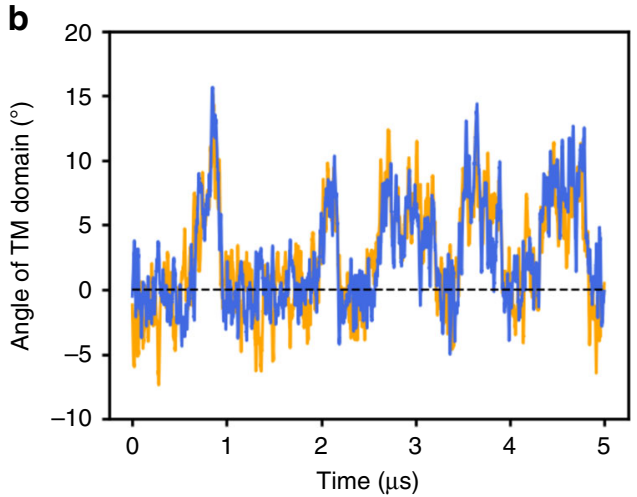

C
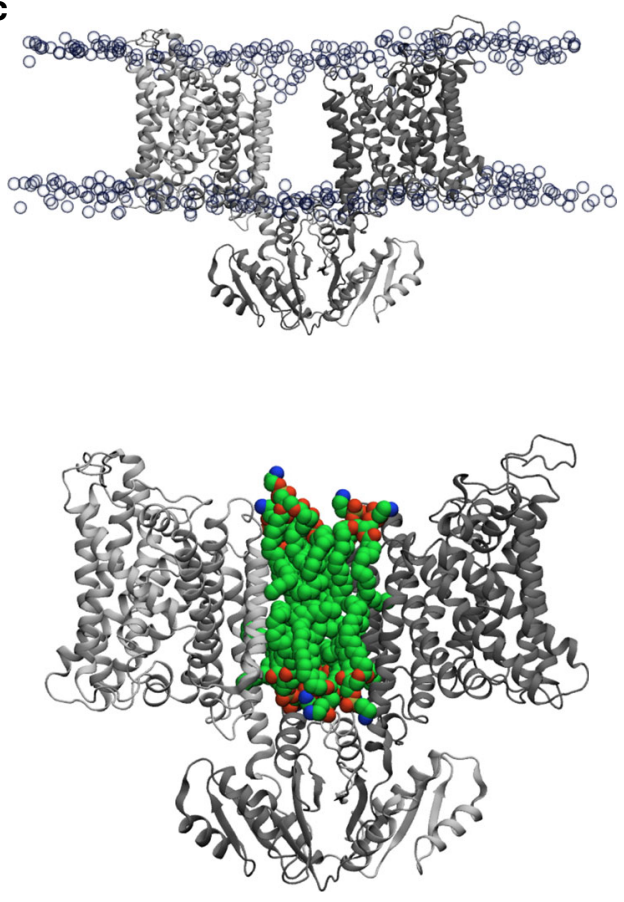

Fig. 3 Molecular dynamics simulations of KimA in a lipid bilayer. a Cut-away views of KimA dimer in a membrane, taken from the CG simulation data. The protein backbone is shown as grey surface and the POPE lipids as green, yellow and blue spheres. $\mathbf{b}$ Representative plot of the angle between the TM domains over time for the CG simulations; $n=4$ independent simulations (see Supplementary Fig. 5 for full data and a schematic of how the angles were computed). c Upright dimer position as determined using $2 \mu$ s of fully atomistic simulation. Protein shown as grey cartoon, lipid phosphates as semitransparent blue circles. $n=3$ repeats, with the upright dimer sampled in 2/3. d Views from atomistic simulation showing the lipid molecules (as coloured spheres) present between the KimA monomer.

Generally, the limited resolution of the map and possibly some dynamics of the ions led to comparably broad densities (Supplementary Fig $3 \mathrm{f}$ and g), which left some uncertainties for the assignment of correct potassium ion binding sites. Two $135 \mathrm{~ns}$ atomistic simulations of the dimer in $150 \mathrm{mM} \mathrm{KCl}$ and in the presence of the three bound $\mathrm{K}^{+}$ions were performed to detail the potassium ion binding. The simulations confirm the existence of three ion binding sites (Fig. $4 \mathrm{f}$ and Supplementary Fig. 6). On average, $c a$. 2.3 ions were bound to each monomer at any one time, with the ions dynamically sampling all three sites. Of the residues surrounding the occluded ion, a short minimum distance and high contact (residue- $\mathrm{K}^{+}$distance less than $0.4 \mathrm{~nm}$ for $>75 \%$ of the simulation time) was observed with residues D36, T121 and Y377, while residues Y43 and T230 make either a more dynamic or more long-range contribution to $\mathrm{K}^{+}$coordination. A rather long minimum distance and hardly any contact was observed with S125. Hence, the occluded $\mathrm{K}^{+}$appears to be located close to the residues forming the thin, cytoplasmic gate, while S125, which was the closest residue to the density seen in
cryo-EM, is not directly involved in ion binding. The coordination of the two potassium ions below the thin gate was confirmed by the MD simulations, the minimum distances between the residues and the ions are similar to the distances determined in the structure. Strong contact was found with residues D36 and D117, while the interaction with E233 was dynamic; in both the cryo-EM map and the MD simulations, E233 is too far from the binding sites for an involvement in potassium ion coordination.

Key residues for substrate binding and trans-inhibition. To further evaluate the role of the residues in close proximity to the bound potassium ions, we tested point-mutated KimA variants for their ability to complement growth at $\mathrm{K}^{+}$limitation (Supplementary Figs. 9 and 10). For wild-type KimA a concentration of the half maximal growth $\left(K_{S}\right)$ of $0.09 \mathrm{mM}$ was determined. Of the residues surrounding the occluded ion, variants $\mathrm{D} 36 \mathrm{~A} / \mathrm{N}$ and $\mathrm{Y} 43 \mathrm{~A}$ completely abolished $\mathrm{K}^{+}$uptake, while S125A, T121A and particularly Y377A led to reduced 

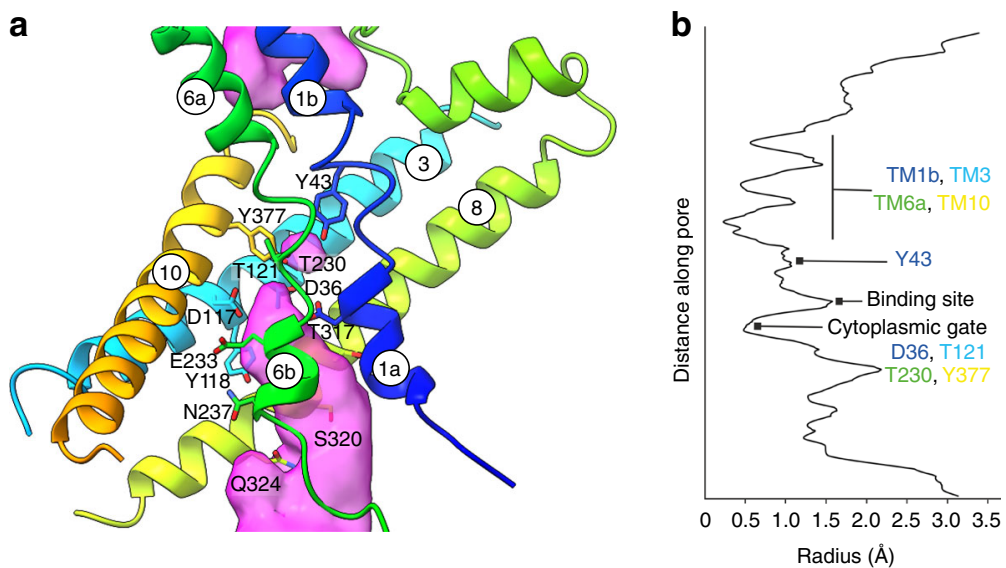

d

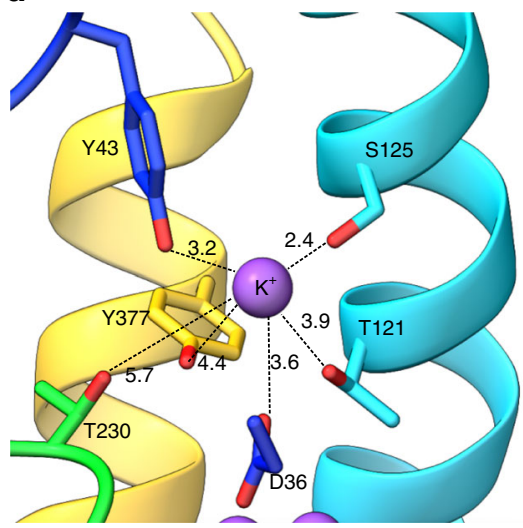

e

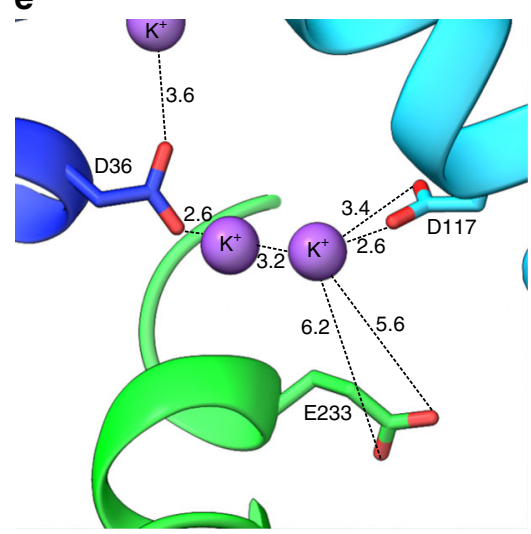

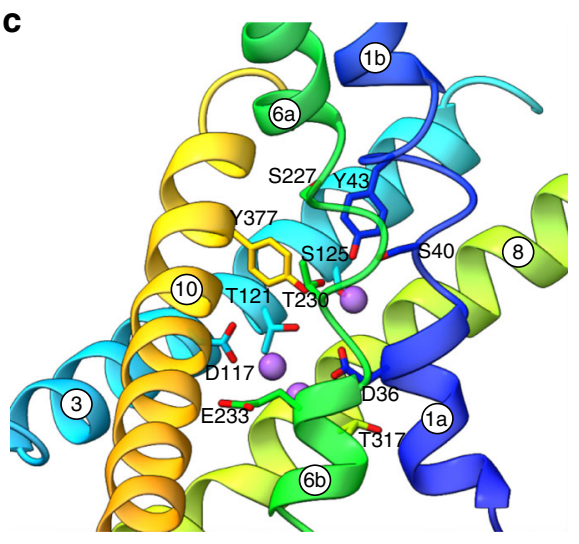

f

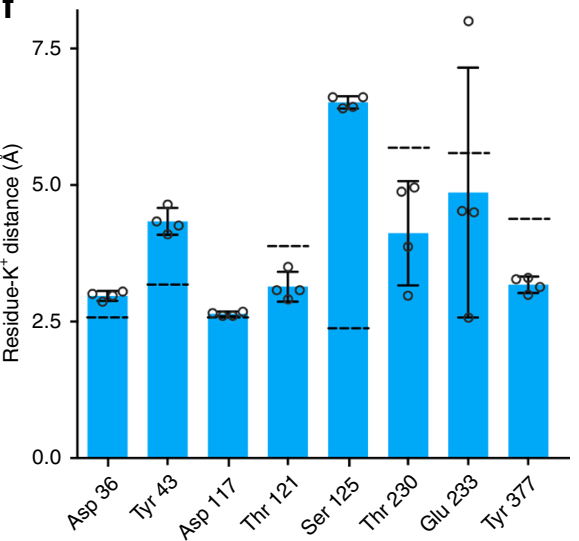

Fig. 4 Structural details of the transmembrane domain. a The translocation pathway reveals a tightly sealed extracellular side, a wide-open intracellular tunnel and the putative occluded potassium ion binding site, which is separated from the cytoplasm by a thin gate. The residues lining the intracellular entrance are represented in sticks. The surface representation of the pathway was calculated with HOLLOW52. $\mathbf{b}$ Pore radii along the translocation pathway of KimA determined by HOLE ${ }^{53}$. Features defining the inward-occluded state are highlighted. c Potential potassium ion binding sites within one

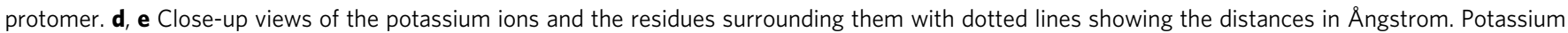
ions are depicted as purple spheres. f Average minimum distance for each residue to the closest $\mathrm{K}^{+}$ion over the course of two $135 \mathrm{~ns}$ atomistic simulations; errors shown are s.d., individual distances as dot plots, $n=4$ repeats. Dashed lines indicate the distances derived from the structural model.

growth rates with $K_{S}$ values of $0.20,0.27$ and $11.0 \mathrm{mM}$, respectively. With a $K_{S}$ of $0.07 \mathrm{mM}$, variant $\mathrm{T} 230 \mathrm{~A}$ enabled a similar growth phenotype as the wild-type protein (Supplementary Fig. 9). The fact that some of these mutations did not simply abolish cell growth but showed a reduction of the apparent affinity corroborates their role in substrate binding and/or occlusion. The effects of mutating residues D36, T121, and Y377 are in agreement with a role in ion coordination. Y43 was suggested to only weakly interact with the potassium ion. Its localization towards the extracellular side of the TM domain suggests that it may instead form the extracellular gate of the binding site, which would explain the lethal effect of mutating it. Residue S125, which does not appear to be directly involved in potassium ion binding, may be important for the overall structure of the binding site and the high affinity binding, while residue T230 is not essential for potassium uptake.

Surprisingly, of the residues near the potassium ions in the cytoplasmic tunnel E233A/Q abolished $\mathrm{K}^{+}$uptake, while D117A/ N/E had no effect on growth (Supplementary Fig. 8). Both the cryo-EM density and the MD simulations suggested that the essential residue E233 is not directly involved in coordinating the bound potassium ions (Fig. 4e, f). However, in the observed inward-facing conformation E233 has a predicted $\mathrm{pK}_{\mathrm{a}}$ of $\sim 8$ and thus could be easily protonated and deprotonated. For that reason, we hypothesized that E233 is responsible for proton coupling. Determining the $\mathrm{K}^{+} / \mathrm{Rb}^{+}$exchange activity of $\mathrm{KimA}_{\mathrm{E} 233 \mathrm{~A}}$ confirmed this assumption: similar to wild-type KimA, the variant could still exchange $\mathrm{K}^{+}$for $\mathrm{Rb}^{+}$, a process uncoupled from proton transport. In contrast, mutation D36A abolished $\mathrm{K}^{+} / \mathrm{Rb}^{+}$exchange by KimA confirming the role of residue D36 in $\mathrm{K}^{+}$binding (Fig. 5a, b, Supplementary Fig. 11). Unlike E233, residue D117 clearly coordinates one of the potassium ions in the cytoplasmic tunnel, and it was therefore surprising that it is not required for transport. A possible explanation is that this residue acts as a sensor for the cytoplasmic potassium ion concentration, preventing further uptake by trans-inhibition. We propose that D117 may have a role in regulation by securing a fast response to variations in potassium concentration. In support of this hypothesis, mutation D117A led to diminished growth of E. coli LB2003 exclusively at high potassium concentration, while cells expressing wild-type KimA grew similar at all tested concentrations (Fig. $5 c$, d). The diminished growth at increased potassium concentrations argues for a toxic effect of an excess of potassium inside the cells as previously described ${ }^{21,30}$. The essential residue D36 coordinates the second potassium ion in the cytoplasmic tunnel as well as the potassium in the actual substrate binding site, and contributes to the thin gate. D36 appears to play a central role in KimA, participating not only in substrate binding and in gating, but also in regulation in a similar way to D117. 
a

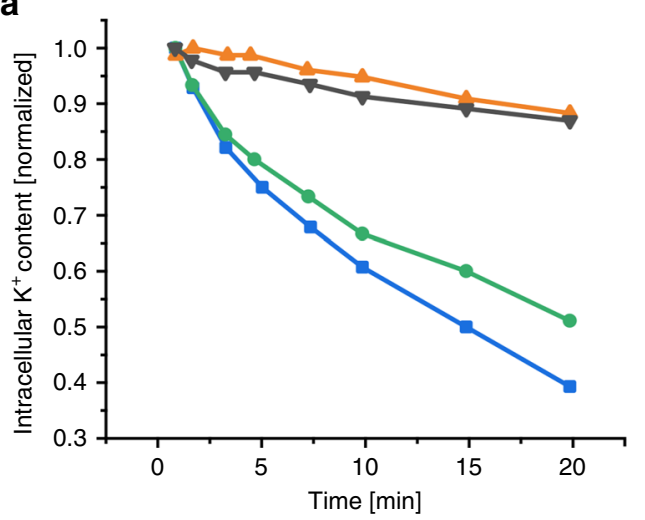

C

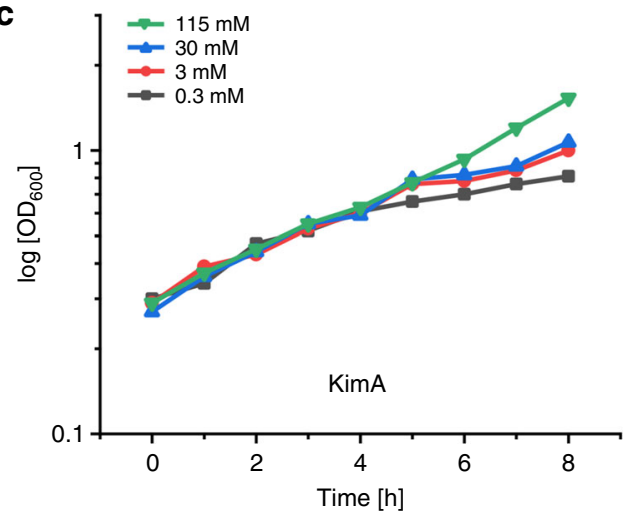

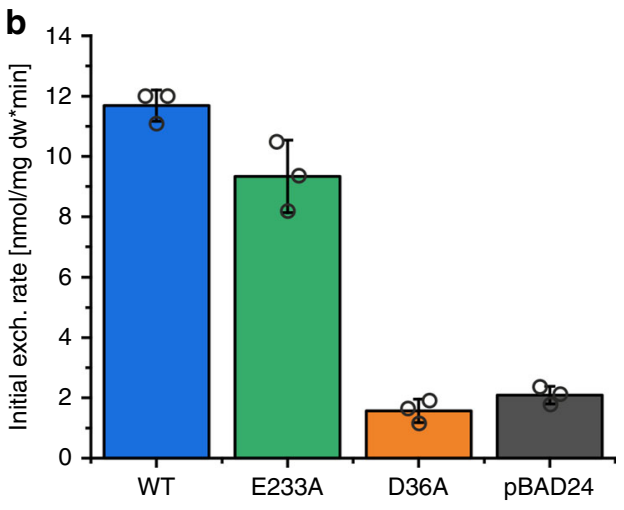

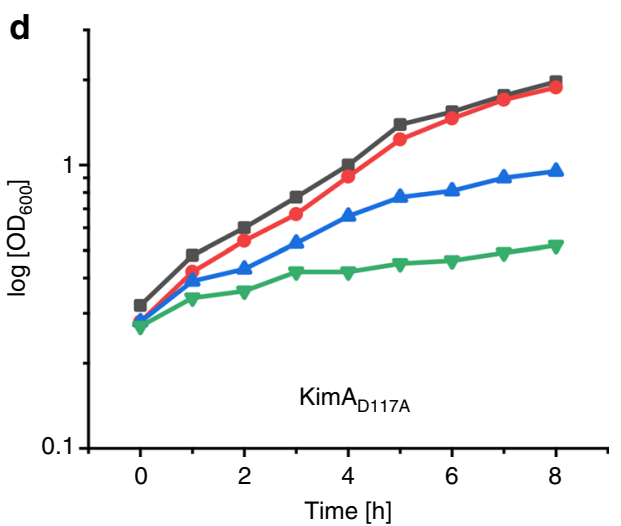

Fig. 5 Involvement of residues in proton coupling or potassium binding or trans-inhibition. a Time courses of the $\mathrm{K}^{+} / \mathrm{Rb}^{+}$exchanges of $E$. coli $\mathrm{LB} 2003$ cells transformed with plasmids encoding wild-type KimA (blue), KimA $A_{E 233 A}$ (green), KimA $A_{D 36 A}$ (orange) or with the empty vector pBAD24 (black). To initiate the exchange, cells loaded with $50 \mathrm{mM} \mathrm{KCl}$ were diluted into an equivalent buffer with $50 \mathrm{mM} \mathrm{RbCl}$. $n=3$ independent experiments; a representative experiment is shown. $\mathbf{b}$ Initial rates of $\mathrm{K}^{+} / \mathrm{Rb}^{+}$exchange given as means of three independent experiments; errors shown are s.d., individual exchange rates as dot plots. c, d Growth curves of E. coli LB2003 expressing wild-type KimA (c) and KimA $A_{D 117 A}$ (d) at different potassium concentrations, ranging from 0.3 to $115 \mathrm{mM} . n=3$ independent experiments; a representative experiment is shown. Source data are provided as a Source Data file.

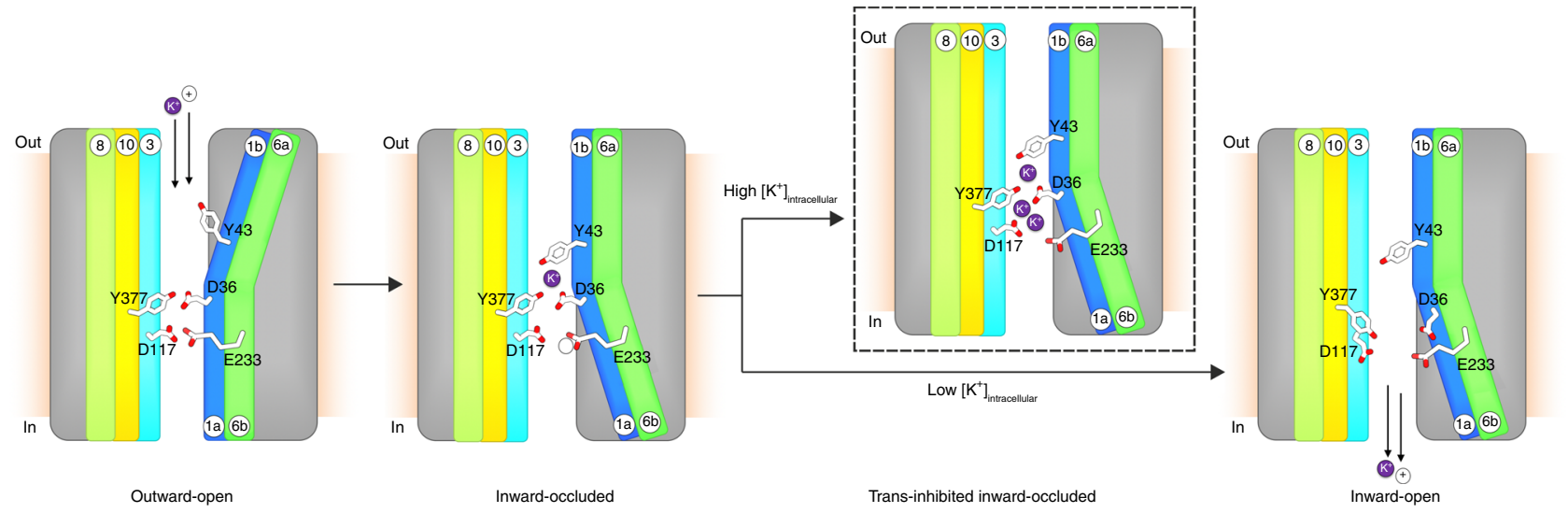

Fig. 6 Schematic representation of alternating access transport in KimA. The outward-open conformation should provide access of protons and potassium ions from the extracellular side to the binding sites, while at the cytoplasmic side the exit tunnel should be tightly sealed. The binding of a proton and a potassium ion could result in the movement of $Y 43$, which serves as external gate occluding the bound substrate. Subsequently, broken helices TM1 and TM6 could alternate, sealing the extracellular tunnel and opening the intracellular tunnel. A thin, intracellular gate formed by residues D36 and Y377 could yet sustain an occluded state. Upon deprotonation of E233, the intracellular gate could open, allowing potassium ion release to the cytoplasm. At high intracellular potassium concentrations, potassium ions could bind within the intracellular tunnel preventing the opening of the intracellular gate. We hypothesize that our cryo-EM structure represents such a trans-inhibited, inward-occluded state (highlighted with dashed box).

\section{Discussion}

Based on the presented data and the knowledge from other APC transporters ${ }^{31,32}$, we propose a model for proton-coupled potassium transport via KimA (Fig. 6). To allow potassium and proton binding from the outside, KimA needs to adopt an outward-open conformation. Therefore, broken helices TM1a and TM6b probably move towards TM helices 3 and 10, tightly sealing the potassium ion binding site from the cytoplasm by main chain 
packing. At the same time, orienting TM1b and TM6a away from TM helices 3 and 10 would open a tunnel to the extracellular space. To completely expose the binding site to the outside, residue Y43 probably reorients, functioning as the extracellular gate. Upon binding of at least one proton and one potassium ion to the respective binding sites (E233 for $\mathrm{H}^{+}, \mathrm{D} 36, \mathrm{~T} 121$ and $\mathrm{Y} 377$ for $\mathrm{K}^{+}$), Y43 could occlude the bound ions from the extracellular side. Subsequently, TM1b and TM6a likely move towards TM helices 3 and 10 tightly sealing the extracellular side, while a displacement of TM1a and TM6b would open the intracellular tunnel. As seen in the presented structure, the potassium ion probably remains occluded towards the intracellular side by the thin intracellular gate formed by D36 and Y377. Ultimately, E233 could get deprotonated and trigger the opening of the intracellular gate, which would expose the binding site to the cytoplasm and allow potassium ion release. We suggest that the herepresented cryo-EM structure represents a trans-inhibited, inwardoccluded state of KimA, in which the intracellular tunnel is filled with potassium ions at high internal potassium concentrations. We propose that these ions prevent the opening of the intracellular gate and the exposure of the potassium ion binding site towards the cytoplasm, hindering the further accumulation of potassium ions despite the presence of a driving proton gradient.

By performing a model-based sequence alignment of KimA with members of the KUP family, we found that the residues we identified as playing an important functional role in KimA are all fully or highly conserved among KUP members, while the sequence conservation in the KUP family is generally low (Supplementary Fig. 12). The overall structure of KimA is in agreement with the predicted overall structure of the low-affinity $\mathrm{K}^{+}$ transporter Kup from E. coli, the best characterized KUP protein $^{11}$. In addition, residues of $E$. coli Kup essential for transport, D23, E116 and E22911 are equivalent to D36, D117, and E233 in KimA (Supplementary Fig. 12). However, the otherwise highly conserved VFGD/IYGD motif within TM helix 1 of KUP members is not conserved in KimA. This leads us to conclude that KimA actually forms a subfamily within the KUP family, and that the presented structure is relevant for all potassium transporters in this family. Our analysis provides the molecular basis for the proton-coupled potassium ion transport in the KUP family and sets the framework for the full characterization of this widely distributed group of transporters, in particular the elucidation of the structural basis for the different potassium ion binding affinities within this family.

\footnotetext{
Methods

Cloning, expression and purification of KimA. The gene encoding KimA was amplified from B. subtilis genomic DNA and cloned into a pBAD24 expression vector including a C-terminal $\mathrm{His}_{10}$-tag. Point mutations in the $\operatorname{kimA}$ gene were applied by restriction-free cloning techniques. A list of all primers used is included in the Supplementary Methods. Wild-type KimA was produced in E. coli strain LB2003 (available from the authors upon request) by growing an overnight culture in KML medium supplemented with $100 \mu \mathrm{g} / \mathrm{mL}$ ampicillin. This culture was diluted 100 -fold in $12 \mathrm{~L}$ of fresh KML medium, and overexpression was induced at an $\mathrm{OD}_{600}$ of 0.8 by the addition of $0.002 \%$ arabinose. After $1.5 \mathrm{~h}$ of induction the cells were harvested and resuspended in $50 \mathrm{mM}$ Tris $\mathrm{pH} 8,100 \mathrm{mM} \mathrm{KCl}, 400 \mathrm{mM}$ $\mathrm{NaCl}, 1 \mathrm{mM}$ EDTA supplemented with $0.5 \mathrm{mM}$ PMSF, $1 \mathrm{mM}$ benzamidine and DNase I. The suspension was passed through a homogenizer at a pressure of $1 \mathrm{kbar}$ to disrupt the cells. The cell extract was centrifuged at $15,000 \times g$ for $15 \mathrm{~min}$ to remove unbroken cells and debris. Membranes were collected by centrifugation at $180,000 \times g$ for $3 \mathrm{~h}$. The membrane pellet was resuspended in solubilization buffer ( $50 \mathrm{mM}$ Tris $\mathrm{pH} 8,100 \mathrm{mM} \mathrm{KCl}, 400 \mathrm{mM} \mathrm{NaCl}$ ) to $50 \mathrm{mg} / \mathrm{mL}$ and solubilized by the addition of 2\% SMA co-polymer Xiran SL30010 P20 (2:1 molar ratio of styrene: maleic acid), provided by Polyscope Polymers B. V., at $4{ }^{\circ} \mathrm{C}$ overnight. Subsequently, the solution was centrifuged at $180,000 \times g$ for $30 \mathrm{~min}$ to remove unsolubilized particles. The supernatant was incubated with Ni-NTA overnight. Then, the resin was washed with 50 column volumes of solubilization buffer supplemented with $50 \mathrm{mM}$ imidazole to remove any unspecific protein. Finally, the protein was eluted using $500 \mathrm{mM}$ imidazole in the solubilization buffer, and further purified by size exclusion chromatography using a Superose $610 / 300$ GL column
}

(GE Healthcare) previously equilibrated with $50 \mathrm{mM}$ Tris pH 8, $100 \mathrm{mM} \mathrm{KCl}$. Fractions containing the protein were pooled and the sample was concentrated to $1 \mathrm{mg} / \mathrm{mL}$ prior to cryo-grid preparation. In the case of the detergent-solubilized KimA, the membrane pellet was solubilized with $1 \%$ DDM (from $20 \%$ stock). For the rest of that purification the buffers were supplemented with $0.04 \%$ DDM.

$\mathbf{K}^{+}$uptake assay. An adapted protocol from ref. ${ }^{33}$ was established for potassium uptake experiments into intact, potassium-depleted E. coli LB2003 cells, which lack all endogenous potassium uptake systems. LB2003 transformed with the plasmid that encodes the protein of interest was grown in $1 \mathrm{~mL}$ KML medium supplemented with $100 \mu \mathrm{g} / \mathrm{mL}$ ampicillin at $37^{\circ} \mathrm{C}$ shaking at $180 \mathrm{rpm}$ overday. The preculture was used to inoculate $50 \mathrm{~mL} \mathrm{~K} 30$ medium (the number indicates the $\mathrm{mM}$ potassium concentration added to the minimal media) supplemented with ampicillin for an overnight culture. The next day, the cells were diluted to an $\mathrm{OD}_{600}$ of 0.15 in $500 \mathrm{~mL} \mathrm{K30} \mathrm{medium} \mathrm{containing} \mathrm{ampicillin} \mathrm{and} 0.002 \% \mathrm{~L}$-arabinose for gene expression. After the cells reached an $\mathrm{OD}_{600}$ of $\sim 0.6-0.8$, they were centrifuged at $5000 \times g$ at $15^{\circ} \mathrm{C}$ for $10 \mathrm{~min}$ and the cell pellets were resuspended in $120 \mathrm{mM}$ Tris- $\mathrm{HCl}$ pH 8 to an $\mathrm{OD}_{600}$ of 30 and washed three times by centrifuging at $4000 \times$ $g$ at $20^{\circ} \mathrm{C}$ for $10 \mathrm{~min}$. After the last centrifugation step, the cells were adjusted to an $\mathrm{OD}_{600}$ of 30 with the same buffer. The resuspended cells were incubated at $37^{\circ} \mathrm{C}$ for $5 \mathrm{~min}$. To release $\mathrm{K}^{+}$and $\mathrm{Na}^{+}$from the cytoplasm, the cells were treated with 1 mM EDTA while gently shaking at $37^{\circ} \mathrm{C}$ for another $5 \mathrm{~min}$. Subsequently, the cells were centrifuged twice to wash out EDTA and ions at $4000 \times g$ at $20^{\circ} \mathrm{C}$ for $8 \mathrm{~min}$ and the pellets were resuspended each time in $100 \mathrm{mM}$ HEPES, $100 \mathrm{mM}$ MES $\mathrm{pH} 7.5$.

For the $\mathrm{K}^{+}$uptake experiment, cells were diluted into flasks to an $\mathrm{OD}_{600}$ of 3 in $100 \mathrm{mM}$ HEPES, $100 \mathrm{mM}$ MES at the desired $\mathrm{pH}$ values under constant shaking at $20{ }^{\circ} \mathrm{C}$. Ten minutes before initiating the potassium uptake, the cells were energized with $0.2 \%$ glycerol and $0.002 \% \mathrm{~L}$-arabinose. The uptake was initiated by addition of various $\mathrm{K}^{+}$concentrations $(0.05 ; 0.1 ; 0.2 ; 0.5 ; 1$ and $2 \mathrm{mM})$. At different time points samples of $1 \mathrm{~mL}$ from each flask were transferred onto $200 \mu \mathrm{L}$ silicone oil with a density of $1.04 \mathrm{~g} / \mathrm{cm}^{3}$ in a $1.5 \mathrm{~mL}$ centrifuge tube. The samples were immediately centrifuged at $17,000 \times g$ at $20^{\circ} \mathrm{C}$ for 2 min to isolate the cells from the supernatant The supernatant and the silicon oil were removed and the cell pellets were cut out with a razor blade and were added to $1 \mathrm{~mL}$ of $5 \%$ trichloracetic acid (TCA) solution. Cell pellets were resuspended by vortexing. The suspensions were frozen at $-20^{\circ} \mathrm{C}$ and subsequently cook at $90^{\circ} \mathrm{C}$ for $10 \mathrm{~min}$ to release the cations. Afterwards the solutions were diluted with $3 \mathrm{~mL} 6.7 \mathrm{mM} \mathrm{CsCl}$ and $4 \mathrm{~mL} 5 \mathrm{mM}$ $\mathrm{CsCl}$. After removing the cell fragments by centrifugation at $4000 \times g$ for $20 \mathrm{~min}$ the $\mathrm{K}^{+}$concentration was determined by flame photometry.

$\mathbf{K}^{+} / \mathbf{R b}^{+}$exchange assay. E. coli LB2003 cells were transformed with plasmids that encode $\mathrm{KimA}, \mathrm{Kim}_{\mathrm{D} 36 \mathrm{~A}}$ and $\mathrm{KimA}_{\mathrm{E} 233 \mathrm{~A}}$, respectively, or with the empty vector pBAD24, and grown in K30 minimal medium as indicated above for the $\mathrm{K}^{+}$ uptake assay. After harvesting, the cell pellets were resuspended and washed twice with $200 \mathrm{mM}$ HEPES-TEA pH 7.5. Afterwards, cells were adjusted to an $\mathrm{OD}_{600}$ of 300 with the same buffer supplemented with $50 \mathrm{mM} \mathrm{KCl}$ and incubated at room temperature for $3 \mathrm{~h}$ to load the cells with potassium ions. To initiate the exchange cells were diluted 100-fold in 200 mM HEPES-TEA pH 7.5 supplemented with 50 $\mathrm{mM} \mathrm{RbCl}$ (or $50 \mathrm{mM} \mathrm{NaCl}$ for the negative control) and with $30 \mu \mathrm{M}$ of the proton ionophore carbonyl cyanide m-chlorophenyl hydrazone (CCCP). CCCP abolished the proton motive force, avoiding active transport. To monitor $\mathrm{K}^{+} / \mathrm{Rb}^{+}$exchange, samples were taken at different time points, cells were treated as described for the $\mathrm{K}^{+}$uptake assay and intracellular $\mathrm{K}^{+}$concentrations were determined using flame photometry.

Complementation assay. Cells harbouring plasmids encoding for KimA and variants thereof were picked from agar KML plates and incubated in $1 \mathrm{~mL} \mathrm{KML}$ medium supplemented with $100 \mu \mathrm{g} / \mathrm{mL}$ ampicillin at $37^{\circ} \mathrm{C}$ shaking at $180 \mathrm{rpm}$ overday. Then, the cells were incubated in $5 \mathrm{~mL} \mathrm{K30} \mathrm{medium} \mathrm{supplemented} \mathrm{with}$ $100 \mu \mathrm{g} / \mathrm{mL}$ ampicillin, at $37^{\circ} \mathrm{C}$ shaking at $180 \mathrm{rpm}$ overnight. The next day, precultures were washed and centrifuged twice at $4000 \times g$ for 10 min in K0 medium to remove external potassium. After that, $5 \mathrm{~mL}$ of different minimal media (K0.01, $\mathrm{K} 0.02, \mathrm{~K} 0.03, \mathrm{~K} 0.05, \mathrm{~K} 0.1, \mathrm{~K} 0.2, \mathrm{~K} 0.5, \mathrm{~K} 1, \mathrm{~K} 3, \mathrm{~K} 10, \mathrm{~K} 30$ and $\mathrm{K} 115)$ each supplemented with $100 \mu \mathrm{g} / \mathrm{mL}$ ampicillin and in the presence of $0.002 \%$ arabinose for gene expression were inoculated to an $\mathrm{OD}_{600}$ of 0.15 . The $\mathrm{OD}_{600}$ was measured hourly for the first $10 \mathrm{~h}$ and after $24 \mathrm{~h}$. After $24 \mathrm{~h}$, samples were taken for SDSPAGE and subsequent Western blotting to analyze the protein production.

Preparation of proteoliposomes. Proteoliposomes were prepared with a standard protocol ${ }^{34}$. Briefly, E. coli polar lipids in chloroform were dried in a rotary evaporator resuspended to $10 \mathrm{mg} / \mathrm{mL}$ and sonicated in a buffer containing $100 \mathrm{mM} \mathrm{NaP}$ i $\mathrm{pH}$ After three freeze-thaw cycles, large unilamellar vesicles were prepared by extrusion through a $400-\mathrm{nm}$ diameter polycarbonate filter. Liposomes were diluted to $4 \mathrm{mg} / \mathrm{mL}$ and destabilized beyond $\mathrm{R}_{\text {sat }}$ with Triton X-100. DDM-solubilized KimA was added to the liposomes at a weight ratio of 1:50 (protein:lipid), and detergent was subsequently removed by the addition of BioBeads. Proteoliposomes were harvested by centrifugation at $250,000 \times g$ and resuspended to a lipid concentration of $10 \mathrm{mg} / \mathrm{mL}$ After three freeze-thaw cycles, proteoliposomes were stored in liquid nitrogen until 
use. For the preparation of the empty control liposomes SEC buffer was added to the lipids in the same ratio as the protein.

Fluorescence-based transport assay. Potassium-dependent proton transport was measured with the $\Delta \mathrm{pH}$-sensitive fluorophore 9-amino-6-chloro-2-methoxyacridine (ACMA). Proteoliposomes and empty liposomes, respectively, were thawed, extruded through a 400-nm polycarbonate filter, centrifuged and resuspended to a final concentration of $10 \mathrm{mg} / \mathrm{mL}$ lipids in $100 \mathrm{mM} \mathrm{NaP} i \mathrm{pH}$ 7. For the fluorescence measurements, proteoliposomes and empty liposomes were diluted 100 -fold into $100 \mathrm{mM} \mathrm{KP}$ i $\mathrm{pH} 7$ and $500 \mathrm{nM}$ ACMA. Fluorescence was recorded at an excitation wavelength of $410 \mathrm{~nm}$ and emission wavelength of $490 \mathrm{~nm}$ in a spectrofluorimeter over time. The $\mathrm{K}^{+}$-coupled $\mathrm{H}^{+}$transport was initiated upon the addition of $500 \mathrm{nM}$ sodium ionophore IV, which initially resulted in a membrane potential of $-120 \mathrm{mV}$ and hindered the establishment of an inhibitory positive potential. At the end of each experiment, the proton ionophore CCCP was added to dissipate the membrane potential allowing protein-independent proton fluxes.

Sequence alignment. Sequence conservation of KimA was evaluated by performing an alignment in T-coffee ${ }^{35}$ using a list of genes obtained from the BLASTNCBI server. Due to the low sequence similarity between KimA and members of the KUP family, a model-based sequence alignment between them was performed using the PROMALS3D ${ }^{36}$ server. In general, the sequence alignment is a progressive method using database searches, secondary structure predictions, and available or provided $3 \mathrm{D}$ structures. For the presented alignments only database searches and secondary structure predictions were applied. The figures were prepared with Jalview ${ }^{37}$.

Negative-staining EM. Samples of KimA solubilized with DDM or SMA copolymer were negatively stained for up to $3 \mathrm{~min}$ with $1 \%$ (w/v) uranyl acetate $\mathrm{pH} 4$. Electron micrographs were acquired with a CCD camera (Gatan Ultrascan 4000) on a Tecnai Spirit at $120 \mathrm{kV}$ under low-dose conditions, at a magnification of $52,000 \times$ for a pixel size at the specimen of $2.11 \AA$. Particles were picked first manually with EMAN boxer ${ }^{38}$ and, after generation of appropriate templates by $2 \mathrm{D}$ classification in Relion $2.1^{39}$, automatically using Gautomatch (by Kai Zhang, MRC-LMB). A total of 3110 and 67,292 good particles of KimA in SMA lipid particles (SMALPs) and DDM, respectively, were picked. Two-dimensional class averages were obtained by $2 \mathrm{D}$ classification with Relion 2.1 .

Cryo-EM specimen preparation and data collection. Cryo-EM grids of KimA in SMALP at $1.1 \mathrm{mg} / \mathrm{mL}$ with $50 \mu \mathrm{M}$ cyclic di-AMP were prepared in a FEI Vitrobot plunge freezer at $10^{\circ} \mathrm{C}$ and $90 \%$ humidity, using Quantifoil R2/2 holey carbon grids (Quantifoil Micro Tools), pre-treated in chloroform for 1 to $2 \mathrm{~h}$ and freshly glowdischarged. The grid was blotted for $9 \mathrm{sec}$ and plunge-frozen in liquid ethane. Images were collected automatically using EPU (Thermo Scientific), on a FEI Titan Krios operating at $300 \mathrm{kV}$ and aligned as previously described ${ }^{40}$, with a Gatan K2 camera in counting mode and with an energy filter. The nominal magnification of $130,000 \mathrm{x}$ yielded a pixel size at the specimen of $1.077 \AA$. Each micrograph was recorded as a movie stack with 40 frames over $8 \mathrm{sec}$, with a calibrated dose of $\sim 1.77 \mathrm{e}^{-} / \AA^{2}$ per frame and defocus values between -0.5 and $-3.2 \mu \mathrm{m}$.

Image processing. A set of 5418 micrographs was collected automatically, of which 4951 were of sufficient quality for processing. Drift correction and dose weighting of each movie stack were performed with MotionCor $2^{41}$. Wholemicrograph CTF was determined with CTFFIND4 ${ }^{42}$ on drift-corrected, non-dose weighted movies. After manual picking of a small particle set using EMAN boxer ${ }^{38}$, templates were generated (first by $2 \mathrm{D}$ classification and later by reprojection of a low-resolution 3D map) for automatic picking by template matching using Gautomatch (by Kai Zhang, MRC-LMB). The initial dataset contained 2,043,209 particles, windowed with a 208 pixels squared box. A low-resolution initial model was generated from $\sim 19,000$ particles using the stochastic gradient descent method implemented in Relion 2.1 and low-pass filtered to $60 \AA^{43}$.

A reference-free 2D classification with ISAC, within Sphire ${ }^{44}$, was used to discard clear false positives and bad particles, outputting a subset with 1,614,900 particle images. A more homogeneous set of 314,399 particles was identified through two consecutive rounds of $3 \mathrm{D}$ classification, with five classes and no symmetry applied, in Relion $3.0^{43}$. After Bayesian polishing, CTF refinement and beam tilt estimation with Relion 3.0, refinement of this subset with a soft mask and applied C2 symmetry produced a map at $3.8 \AA$. However, the resolution degraded towards the periphery of the dimer, especially at the cytoplasmic domain, due to small variations in the relative position of the KimA monomers. Since the signal from a single monomer was insufficient for proper alignment, we attempted to identify a particle subset where these deviations were minimized through a $3 \mathrm{D}$ classification of the symmetry-expanded dataset (629,798 particles) with three classes, only local searches and a reference map low-pass filtered to $4.5 \AA$; after every five iterations, the map of each class was aligned to a partial map, composed of one transmembrane domain and one cytoplasmic domain, within Chimera ${ }^{45}$, in order to keep the position of one half of the dimer constant, while the position of the other half drove the classification (a similar approach was implemented in ref. ${ }^{46}$ ). The best 3D class contained 198,366 particles. Symmetry expansion was reversed by removal of duplicates, for a total of 149,724 unique particle images. A homogeneous refinement of these particles with cryoSPARC $2^{47}$ produced a map with a nominal resolution of $3.7 \AA$ and improved densities at the periphery. Local resolution was estimated with cryoSPARC $2^{47}$.

Model building and validation. An homology model of the transmembrane domain of KimA obtained by the Phyre2 server ${ }^{48}$ was docked initially into the cryo-EM map using USCF Chimera ${ }^{45}$ and used as a starting point for modelling with $\mathrm{Coot}^{49}$. The cytoplasmic domain was built de novo. The model was then subjected to an iterative process of real space refinement using Phenix.real_space_refinement ${ }^{50}$ with geometry and secondary structure restraints followed by manual inspection and adjustments in $\operatorname{Coot}^{49}$. The final model includes 572 residues of the 607 that compose a KimA monomer, lacking the first 26 amino acid residues at the $\mathrm{N}$ terminus and residues $480-481,534-538$ and 607 in the cytoplasmic domain. The geometries of the atomic model were evaluated by MolProbity ${ }^{51}$. The translocation pathway and the surface representation were obtained with HOLLOW ${ }^{52}$. Pore radii along the translocation pathway were calculated using HOLE software ${ }^{53} \cdot \mathrm{pK}_{\mathrm{a}}$ calculations were performed with the multiconformation continuum electrostatics (MCCE) program ${ }^{54}$. Figures were prepared with UCSF ChimeraX ${ }^{55}$ and PyMOL.

CG simulations. CG simulations of dimeric KimA were run using the CG Martini forcefield ${ }^{23,24}$, using the open beta 3.0.b.3.2 version. Additional bonds of $500 \mathrm{~kJ} \mathrm{~mol}^{-1}$ $\mathrm{nm}^{-2}$ were applied between all protein backbone beads within $1 \mathrm{~nm}$, except for interactions across the extracellular interface of the TM domains, designed to allow inter-domain dynamics and not bias the simulations towards the cryo-EM pose.

Initial simulations were run following the MemProtMD protocol ${ }^{56,57}$. The input protein was aligned accordingly on the $x y$ plane, and POPE (1-palmitoyl-2-oleoylsn-glycero-3-phosphoethanolamine) lipids were placed randomly around the transmembrane region of protein, in a $z$ range of $8 \mathrm{~nm}$. All systems were solvated with Martini waters and $\mathrm{Na}^{+}$and $\mathrm{Cl}^{-}$ions to a neutral charge and $0.15 \mathrm{M}$. Selfassembly simulations were run to allow the membrane lipids to assemble into a bilayer around the protein. These were minimized using the steepest descents methods, then run over $100 \mathrm{~ns}$ in the NPT ensemble, with V-rescale temperature coupling 58 at $323 \mathrm{~K}$ and semi-isotropic Berendsen pressure coupling at $1 \mathrm{bar}^{59}$, with positional restraints of $1000 \mathrm{~kJ} \mathrm{~mol}^{-1} \mathrm{~nm}^{-2}$ applied to the backbone beads.

Following this, the systems were simulated for a further $4 \times 5 \mu$ s, using V-rescale temperature coupling at $323 \mathrm{~K}$ and a semi-isotropic Parrinello-Rahman barostat ${ }^{60}$. Electrostatics were described using the reaction field method, with a cut-off of 1.1 $\mathrm{nm}$ using a potential shift modifier, and van der Waals interactions were shifted from 0.9-1.1 nm. Bonds were constrained using the LINCS algorithm. All simulations were run using Gromacs $2019^{61}$. Data were analyzed in Gromacs ${ }^{62}$ or $\mathrm{VMD}^{63}$, as described in the figure legends.

Atomistic simulation. Snapshots of the KimA dimer following $100 \mathrm{~ns}$ of restrained CG simulation in a POPC bilayer were converted to the CHARMM36 forcefield ${ }^{64}$ following the CG2AT protocol ${ }^{65}$. Systems were solvated with TIP3P water and $\mathrm{Na}^{+}$ and $\mathrm{Cl}^{-}$ions to $0.15 \mathrm{M}$. Electrostatics were handled using the Particle-Mesh-Ewald method, and a force-switch modifier was applied to the Van der Waals forces. Dispersion corrections were turned off. Systems were equilibrated for $2.5 \mathrm{~ns}$ with protein backbone restraints, before production simulations were run with V-rescale temperature coupling at $310 \mathrm{~K}$ using a time constant of $0.1 \mathrm{ps}$ and ParrinelloRahman semi-isotropic pressure coupling of 1 bar with a time constant of $2 \mathrm{ps,}$ using 4 fs time steps with virtual-sites on the protein and lipids ${ }^{66}$. All simulations were run in Gromacs $2019^{62}$.

For analyses of dimer stability, three simulations were run for ca. $2 \mu$ s to allow for the slower kinetics of dimer rearrangement to occur. For $\mathrm{K}^{+}$ion binding analysis, the $\mathrm{K}^{+}$ions of the structural model were reintroduced to the binding sites, and the system was re-solvated with $\mathrm{K}^{+}$and $\mathrm{Cl}^{-}$ions to $0.15 \mathrm{M}$. The bound ions and protein backbone were restrained for $1 \mathrm{~ns}$ of equilibration, before two production simulations of $135 \mathrm{~ns}$ were run, for a total of $n=4$ ion binding sites.

Reporting summary. Further information on research design is available in the Nature Research Reporting Summary linked to this article.

\section{Data availability}

Data supporting the findings of this manuscript are available from the corresponding authors upon reasonable request. A reporting summary for this Article is available as a Supplementary Information file. The source data underlying Figs. 1 and 5 and Supplementary Figs. 9 and 11 are provided as a Source Data file. The cryo-EM map and the model were deposited in the wwPDB with accession codes EMD-10092 and 6S3K, respectively.

Received: 22 August 2019; Accepted: 10 January 2020; Published online: 31 January 2020 


\section{References}

1. Durell, S. R. \& Guy, H. R. Structural models of the KtrB, TrkH, and Trk1,2 symporters based on the structure of the KcsA K(+) channel. Biophys. J. 77, 789-807 (1999).

2. Bossemeyer, D., Schlösser, A. \& Bakker, E. P. Specific cesium transport via the Escherichia coli Kup (TrkD) $\mathrm{K}^{+}$uptake system. J. Bacteriol. 171, 2219-2221 (1989).

3. Quintero, F. J. \& Blatt, M. R. A new family of $\mathrm{K}^{+}$transporters from Arabidopsis that are conserved across phyla. FEBS Lett. 415, 206-211 (1997).

4. Santa-Maria, G. E., Rubio, F., Dubcovsky, J. \& Rodriguez-Navarro, A. The HAK1 gene of barley is a member of a large gene family and encodes a highaffinity potassium transporter. Plant Cell 9, 2281-2289 (1997).

5. Liu, Y. et al. Potassium transport of Salmonella is important for type III secretion and pathogenesis. Microbiology 159, 1705-1719 (2013).

6. Ochrombel, I., Ott, L., Krämer, R., Burkovski, A. \& Marin, K. Impact of improved potassium accumulation on $\mathrm{pH}$ homeostasis, membrane potential adjustment and survival of Corynebacterium glutamicum. Biochim. Biophys. Acta 1807, 444-450 (2011).

7. Gries, C. M., Bose, J. L., Nuxoll, A. S., Fey, P. D. \& Bayles, K. W. The Ktr potassium transport system in Staphylococcus aureus and its role in cell physiology, antimicrobial resistance and pathogenesis. Mol. Microbiol. 89, 760-773 (2013).

8. Price-Whelan, A. et al. Transcriptional profiling of Staphylococcus aureus during growth in $2 \mathrm{M} \mathrm{NaCl}$ leads to clarification of physiological roles for Kdp and Ktr $\mathrm{K}^{+}$uptake systems. MBio 4, https://doi.org/10.1128/mBio.00407-13 (2013)

9. Vastermark, A., Wollwage, S., Houle, M. E., Rio, R. \& Saier, M. H. Jr. Expansion of the APC superfamily of secondary carriers. Proteins $\mathbf{8 2}$ 2797-2811 (2014).

10. Banuelos, M. A., Klein, R. D., Alexander-Bowman, S. J. \& Rodriguez-Navarro, A. A potassium transporter of the yeast Schwanniomyces occidentalis homologous to the Kup system of Escherichia coli has a high concentrative capacity. EMBO J. 14, 3021-3027 (1995).

11. Sato, Y. et al. Defining membrane spanning domains and crucial membrane-localized acidic amino acid residues for $\mathrm{K}(+)$ transport of a Kup/HAK/KT-type Escherichia coli potassium transporter. J. Biochem. 155, 315-323 (2014).

12. Nanatani, K. et al. Comparative analysis of kdp and ktr mutants reveals distinct roles of the potassium transporters in the model cyanobacterium Synechocystis sp. strain PCC 6803. J. Bacteriol. 197, 676-687 (2015).

13. Santa-Maria, G. E., Oliferuk, S. \& Moriconi, J. I. KT-HAK-KUP transporters in major terrestrial photosynthetic organisms: a twenty years tale. J. Plant Physiol. 226, 77-90 (2018).

14. Rodriguez-Navarro, A., Blatt, M. R. \& Slayman, C. L. A potassium-proton symport in Neurospora crassa. J. Gen. Physiol. 87, 649-674 (1986).

15. Trchounian, A. \& Kobayashi, H. Kup is the major $\mathrm{K}^{+}$uptake system in Escherichia coli upon hyper-osmotic stress at a low pH. FEBS Lett. 447, 144-148 (1999).

16. Scherzer, S. et al. Calcium sensor kinase activates potassium uptake systems in gland cells of Venus flytraps. Proc. Natl Acad. Sci. USA 112, 7309-7314 (2015).

17. Senn, M. E., Rubio, F., Banuelos, M. A. \& Rodriguez-Navarro, A. Comparative functional features of plant potassium HvHAK1 and HvHAK2 transporters. J. Biol. Chem. 276, 44563-44569 (2001).

18. Mangano, S., Silberstein, S. \& Santa-Maria, G. E. Point mutations in the barley HvHAK1 potassium transporter lead to improved $\mathrm{K}^{+}$-nutrition and enhanced resistance to salt stress. FEBS Lett. 582, 3922-3928 (2008).

19. Aleman, $\mathrm{F}$. et al. The $\mathrm{F} 130 \mathrm{~S}$ point mutation in the Arabidopsis high-affinity $\mathrm{K}$ $(+)$ transporter AtHAK5 increases $\mathrm{K}(+)$ over $\mathrm{Na}(+)$ and $\mathrm{Cs}(+)$ selectivity and confers $\mathrm{Na}(+)$ and $\mathrm{Cs}(+)$ tolerance to yeast under heterologous expression. Front Plant Sci. 5, 430 (2014).

20. Han, M., Wu, W., Wu, W. H. \& Wang, Y. Potassium transporter KUP7 is involved in $\mathrm{K}(+)$ acquisition and translocation in Arabidopsis root under $\mathrm{K}$ (+)-limited conditions. Mol. Plant 9, 437-446 (2016).

21. Gundlach, J. et al. Control of potassium homeostasis is an essential function of the second messenger cyclic di-AMP in Bacillus subtilis. Sci. Signal 10, https:// doi.org/10.1126/scisignal.aal3011 (2017).

22. Hellwig, N. et al. Native mass spectrometry goes more native: investigation of membrane protein complexes directly from SMALPs. Chem. Commun. 54, 13702-13705 (2018).

23. Marrink, S. J., Risselada, H. J., Yefimov, S., Tieleman, D. P. \& de Vries, A. H. The MARTINI force field: coarse grained model for biomolecular simulations. J. Phys. Chem. B 111, 7812-7824 (2007).

24. Monticelli, L. et al. The MARTINI coarse-grained force field: extension to proteins. J. Chem. Theory Comput 4, 819-834 (2008).

25. Izard, T. The crystal structures of phosphopantetheine adenylyltransferase with bound substrates reveal the enzyme's catalytic mechanism. J. Mol. Biol. 315, 487-495 (2002).
26. Warmuth, S., Zimmermann, I. \& Dutzler, R. X-ray structure of the C-terminal domain of a prokaryotic cation-chloride cotransporter. Structure 17, 538-546 (2009).

27. Gundlach, J. et al. Sustained sensing in potassium homeostasis: Cyclic di-AMP controls potassium uptake by KimA at the levels of expression and activity. J. Biol. Chem., https://doi.org/10.1074/jbc.RA119.008774 (2019).

28. Shi, Y. Common folds and transport mechanisms of secondary active transporters. Annu Rev. Biophys. 42, 51-72 (2013).

29. Krishnamurthy, H., Piscitelli, C. L. \& Gouaux, E. Unlocking the molecular secrets of sodium-coupled transporters. Nature 459, 347-355 (2009).

30. Hänelt, I. et al. Gain of function mutations in membrane region $\mathrm{M} 2 \mathrm{C} 2$ of $\mathrm{KtrB}$ open a gate controlling $\mathrm{K}^{+}$transport by the KtrAB system from Vibrio alginolyticus. J. Biol. Chem. 285, 10318-10327 (2010).

31. “'Jungnickel, K. E. J., Parker, J. L. \& Newstead, S. Structural basis for amino acid transport by the CAT family of SLC7 transporters. Nat. Commun. 9, 550 (2018).

32. Ma, J. et al. Structural basis for substrate binding and specificity of a sodiumalanine symporter AgcS. Proc. Natl Acad. Sci. USA 116, 2086-2090 (2019).

33. Bakker, E. P. \& Mangerich, W. E. Interconversion of components of the bacterial proton motive force by electrogenic potassium transport. J. Bacteriol. 147, 820-826 (1981).

34. Geertsma, E. R., Nik Mahmood, N. A., Schuurman-Wolters, G. K. \& Poolman, $B$. Membrane reconstitution of $\mathrm{ABC}$ transporters and assays of translocator function. Nat. Protoc. 3, 256-266 (2008).

35. Notredame, C., Higgins, D. G. \& Heringa, J. T-Coffee: a novel method for fast and accurate multiple sequence alignment. J. Mol. Biol. 302, 205-217 (2000).

36. Pei, J., Kim, B. H. \& Grishin, N. V. PROMALS3D: a tool for multiple protein sequence and structure alignments. Nucleic Acids Res. 36, 2295-2300 (2008)

37. Waterhouse, A. M., Procter, J. B., Martin, D. M., Clamp, M. \& Barton, G. J. Jalview Version 2-a multiple sequence alignment editor and analysis workbench. Bioinformatics 25, 1189-1191 (2009).

38. Ludtke, S. J., Baldwin, P. R. \& Chiu, W. EMAN: semiautomated software for high-resolution single-particle reconstructions. J. Struct. Biol. 128, 82-97 (1999).

39. Scheres, S. H. RELION: implementation of a Bayesian approach to cryo-EM structure determination. J. Struct. Biol. 180, 519-530 (2012).

40. Mills, D. J., Vitt, S., Strauss, M., Shima, S. \& Vonck, J. De novo modeling of the $\mathrm{F}(420)$-reducing $[\mathrm{NiFe}]$-hydrogenase from a methanogenic archaeon by cryoelectron microscopy. Elife 2, e00218 (2013).

41. Zheng, S. Q. et al. MotionCor2: anisotropic correction of beam-induced motion for improved cryo-electron microscopy. Nat. Methods 14, 331-332 (2017).

42. Rohou, A. \& Grigorieff, N. CTFFIND4: Fast and accurate defocus estimation from electron micrographs. J. Struct. Biol. 192, 216-221 (2015).

43. Zivanov, J. et al. New tools for automated high-resolution cryo-EM structure determination in RELION-3. Elife 7, https://doi.org/10.7554/eLife.42166 (2018).

44. Moriya, T. et al. High-resolution single particle analysis from electron cryomicroscopy images using SPHIRE. JoVE, e55448, https://doi.org/10.3791/ 55448 (2017).

45. Pettersen, E. F. et al. UCSF Chimera-a visualization system for exploratory research and analysis. J. Comput. Chem. 25, 1605-1612 (2004).

46. Zhang, C. et al. Analysis of discrete local variability and structural covariance in macromolecular assemblies using Cryo-EM and focused classification. Ultramicroscopy 203, 170-180 (2019).

47. Punjani, A., Rubinstein, J. L., Fleet, D. J. \& Brubaker, M. A. cryoSPARC: algorithms for rapid unsupervised cryo-EM structure determination. Nat. Methods 14, 290-296 (2017).

48. Kelley, L. A., Mezulis, S., Yates, C. M., Wass, M. N. \& Sternberg, M. J. The Phyre2 web portal for protein modeling, prediction and analysis. Nat. Protoc. 10, 845-858 (2015)

49. Emsley, P. \& Cowtan, K. Coot: model-building tools for molecular graphics. Acta Crystallogr. D. Biol. Crystallogr. 60, 2126-2132 (2004).

50. Adams, P. D. et al. PHENIX: building new software for automated crystallographic structure determination. Acta Crystallogr. D. Biol. Crystallogr. 58, 1948-1954 (2002).

51. Chen, V. B. et al. MolProbity: all-atom structure validation for macromolecular crystallography. Acta Crystallogr. D. Biol. Crystallogr. 66, 12-21 (2010).

52. Ho, B. K. \& Gruswitz, F. HOLLOW: generating accurate representations of channel and interior surfaces in molecular structures. BMC Struct. Biol. 8, 49 (2008).

53. Smart, O. S., Neduvelil, J. G., Wang, X., Wallace, B. A. \& Sansom, M. S. HOLE: a program for the analysis of the pore dimensions of ion channel structural models. J. Mol. Graph 14, 354-360 (1996). 376.

54. Georgescu, R. E., Alexov, E. G. \& Gunner, M. R. Combining conformational flexibility and continuum electrostatics for calculating $\mathrm{pK}(\mathrm{a}) \mathrm{s}$ in proteins. Biophys. J. 83, 1731-1748 (2002).

55. Goddard, T. D. et al. UCSF ChimeraX: Meeting modern challenges in visualization and analysis. Protein Sci. 27, 14-25 (2018). 
56. Stansfeld, P. J. et al. MemProtMD: automated insertion of membrane protein structures into explicit lipid membranes. Structure 23, 1350-1361 (2015).

57. Newport, T. D., Sansom, M. S. P. \& Stansfeld, P. J. The MemProtMD database: a resource for membrane-embedded protein structures and their lipid interactions. Nucleic Acids Res. 47, D390-D397 (2019).

58. Bussi, G., Donadio, D. \& Parrinello, M. Canonical sampling through velocity rescaling. J. Chem. Phys. 126, 014101 (2007).

59. Berendsen, H. J. C., Postma, J. P. M., Vangunsteren, W. F., Dinola, A. \& Haak, J. R. Molecular-dynamics with coupling to an external bath. J. Chem. Phys. 81, 3684-3690 (1984).

60. Parrinello, M. \& Rahman, A. Polymorphic transitions in single-crystals - a new molecular-dynamics method. J. Appl. Phys. 52, 7182-7190 (1981).

61. Van Der Spoel, D. et al. GROMACS: fast, flexible, and free. J. Comput. Chem. 26, 1701-1718 (2005).

62. Berendsen, H. J. C., Vanderspoel, D. \& Vandrunen, R. Gromacs - a messagepassing parallel molecular-dynamics implementation. Comput. Phys. Commun. 91, 43-56 (1995).

63. Humphrey, W., Dalke, A. \& Schulten, K. VMD: visual molecular dynamics. J. Mol. Graph 14, 33-38 (1996). 27-38.

64. Huang, J. \& MacKerell, A. D. Jr. CHARMM36 all-atom additive protein force field: validation based on comparison to NMR data. J. Comput. Chem. 34, 2135-2145 (2013).

65. Stansfeld, P. J. \& Sansom, M. S. From coarse grained to atomistic: a serial multiscale approach to membrane protein simulations. J. Chem. Theory Comput. 7, 1157-1166 (2011).

66. Olesen, K., Awasthi, N., Bruhn, D. S., Pezeshkian, W. \& Khandelia, H. Faster Simulations with a $5 \mathrm{fs}$ Time Step for Lipids in the CHARMM Force Field. J. Chem. Theory Comput. 14, 3342-3350 (2018).

\section{Acknowledgements}

We thank Werner Kühlbrandt for his support of the cryo-EM. Jörg Stülke is acknowledged for helpful discussions about KimA, Arne Möller for helpful discussions on image processing, Mark Sansom for fruitful discussions on the MD simulations and Ahmadreza Mehdipour for assistance with $\mathrm{pK}_{\mathrm{a}}$ calculations. We thank Irfan Alibay and Owen Vickery for research software engineering support and implementation of lipid virtual sites for MD. Polyscope Polymers B. V. is acknowledged for providing SMA co-polymer Xiran SL30010 P20. This work was supported by the Max Planck Society (J.V.) and by the German Research Foundation via the Cluster of Excellence Frankfurt (Macromolecular Complexes) (I.H.). P.J.S. and R.A.C. were funded by Wellcome [208361/Z/17/ Z]. Research in P.J.S.'s lab is funded by the MRC (MR/S009213/1) and BBSRC (BB/ P01948X/1, BB/R002517/1 BB/S003339/1). This project made use of time on ARCHER granted via the UK High-End Computing Consortium for Biomolecular Simulation, HECBioSim (http://hecbiosim.ac.uk), supported by EPSRC (grant no. EP/R029407/ 1), Athena at HPC Midlands+, which is supported by the EPSRC (EP/P020232/1), and the University of Warwick Scientific Computing Research Technology Platform.

\section{Author contributions}

I.T. and I.H. conceived the project. I.T. cloned, expressed and purified KimA wild-type and variants. I.T. and I.H. designed the functional experiments. I.T., D.G., N.A. and V.M. performed the functional experiments. D.J.M. and J.S.S. optimized the high-resolution EM alignment and the data collection procedure. J.S.S. prepared the samples for cryo-EM analysis, and collected and processed the data. I.T. and J.S.S. built and validated the atomic model. R.A.C. and P.J.S. performed the MD simulations. I.T., J.S.S., R.A.C., P.J.S., J.V. and I.H. interpreted the data. I.T. and J.S.S. wrote the initial draft and I.T., J.S.S., R.A. C., P.J.S., J.V. and I.H. wrote the manuscript. P.J.S., J.V. and I.H. supervised work, and P. J.S. and I.H. acquired funding.

\section{Competing interests}

The authors declare no competing interests.

\section{Additional information}

Supplementary information is available for this paper at https://doi.org/10.1038/s41467020-14441-7.

Correspondence and requests for materials should be addressed to J.V. or I.H.

Peer review information Nature Communications thanks Show-Ling Shyng, Nobuyuki Uozumi and the other, anonymous, reviewer(s) for their contribution to the peer review of this work. Peer reviewer reports are available.

Reprints and permission information is available at http://www.nature.com/reprints

Publisher's note Springer Nature remains neutral with regard to jurisdictional claims in published maps and institutional affiliations.

(c) Open Access This article is licensed under a Creative Commons Attribution 4.0 International License, which permits use, sharing, adaptation, distribution and reproduction in any medium or format, as long as you give appropriate credit to the original author(s) and the source, provide a link to the Creative Commons license, and indicate if changes were made. The images or other third party material in this article are included in the article's Creative Commons license, unless indicated otherwise in a credit line to the material. If material is not included in the article's Creative Commons license and your intended use is not permitted by statutory regulation or exceeds the permitted use, you will need to obtain permission directly from the copyright holder. To view a copy of this license, visit http://creativecommons.org/ licenses/by/4.0/.

(C) The Author(s) 2020 